\title{
A NOTE ON THE HERMITE-HADAMARD INEQUALITY
}

\author{
XIANG GAO
}

Abstract. In this note we give a new generalization of the well-known Hermite-Hadamard inequality

Mathematics subject classification (2010): 52A40, 52A41.

Keywords and phrases: Convex functions, Hermite-Hadamard inequality, Jensen's inequality.

\section{REFERENCES}

[1] J. HADAMARD, Étude sur les propriétés des fonctions entiéres et en particulier d'une fonction considérée par Riemann, J. Math. Pures Appl., 58 (1893), 171-215.

[2] D. S. Mitrinović And I. B. Lacković, Hermite and Convexity, Aequationes Math., 28 (1985), 229-232.

[3] C. Niculescu And L.-E. Persson, Old and New on the Hermite-Hadamard inequality, Real Analysis Exchange, 2004.

[4] S. S. Dragomir And C. E. M. Pearce, Selected Topics on Hermite-Hadamard Inequalities, (RGMIA Monographs http://rgmia.vu.edu.au /monographs/hermitehadamard.html), Victoria University, 2000.

[5] A. El Farissi, Simple Proof and Refinement of Hermite-Hadamard Inequality, J. Math. Inequal, preprint.

[6] G. H. Hardy, J. E. Littlewood and G. Polya, Inequalities, Cambridge University Press, Cambridge, MA, 1952. 